\title{
The Effect of Dwell Time to Creep-Fatigue Crack Growth
}

\author{
Wen $\mathrm{Li}^{1,}$, , Guobin Zhang ${ }^{2, \mathrm{~b}}$, Xiaohong Yang ${ }^{1}$, Yan $\mathrm{Jiao}^{1}$ \\ ${ }^{1}$ Beijing Institute Of Control Engineering, Beijing, 100190, China \\ ${ }^{2}$ Beijing electric vehicle Co., Itd, Beijing, 102606, China

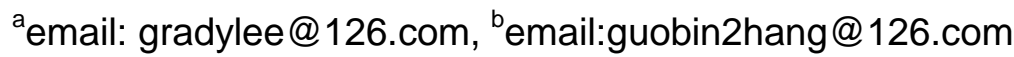

Keywords: Creep damage evolution; Fatigue damage evolution; Hold time effects

\begin{abstract}
In engineering application a quantitative description of both fatigue and creep damage mechanisms is demanded for high-temperature alloy. The present work employs the cohesive zone model to numerically simulate the crack propagation in a nickel-based super-alloy. Effect of loading hold period is discussed. The comparison shows a reasonable agreement between numerical results and experimental observations.
\end{abstract}

\section{Introduction}

Many mechanical parts are working under the various external loading conditions, suffering from thermal, mechanical and environmental loads. Tong et al. [1] investigated effects of creep, fatigue and oxidation on crack propagation in a nickel-based super-alloy at $650^{\circ} \mathrm{C}$. The work concluded that the oxidation appears to be the predominant mechanism for crack growth under long dwell loading condition due to the limited creep at the crack tip. Lu et al. [2] investigated the influence of hold time on creep-fatigue crack growth behavior. Several different hold time levels were employed in the test. The results shown that hold time has positive effect on the crack growth. To give a more accurate prediction, especially for complex geometry parts, a constitutive description of the creep fatigue crack is of importance.

In mechanical product design, the finite element method (FEM) is becoming a staple and usableness numerical analysis tool. Nowadays, more and more attentions are focused on the cohesive zone model (CZM). The earliest concept of the cohesive zone modeling was proposed by Barenblatt [3] to research ideal brittle materials. However, modeling of creep fatigue is still a challenging task for computational mechanics of materials.

The aim of the present work is to find out the effect of dwell time on the crack growth in nickel-based PM super-alloy in the cohesive zone model.

\section{Cohesive zone models}

\subsection{Modeling of fatigue crack growth}

For creep fatigue crack problems, the material damage consists of both cyclic mechanical damage, $D_{f}$, and creep damage, $D_{c}$, that is

$$
D=D_{f}+D_{c}
$$

Considering the cyclic loading, the damage variable, $D_{f}$, is introduced to describe the cyclic damage accumulation in the cohesive zone model. The description of $D_{f}$ is the key issue in the cyclic material damage modeling. In the past years various suggestions were published. $\mathrm{Xu}$ and Yuan [4] have given out of a model modified from the model of Roe and Siegmund [5],

$$
\dot{D}_{f}=\frac{\left|\dot{\kappa}_{n}\right|}{d_{\Sigma}}\left(\frac{T_{n}}{\sigma_{\max , 0}}+1\right)^{m} H\left(\sigma_{e q}-f_{0}\right)
$$

Where $\sigma_{e q}$ and $f_{0}$ are the equivalent principal stress around the cohesive zone tip and the 
material fatigue limit, respectively.

\subsection{Creep damage accumulation}

At high temperature, the material mainly suffers from thermal attack. If the creep damage is introduced into the cohesive zone model, following the suggestion in [6], the creep damage evolution in the cohesive zone model can be written as,

$$
\dot{D}_{c}=\left(\frac{\|T\|-(1-D) T_{c}}{C}\right)^{r}(1-D)^{-P}
$$

Where $C, p$ and $r$ are material parameters which can be determined with experimental data. In order to explicitly consider effects of the temperature, an additional term related to the temperature $(\vartheta$ in $\mathrm{K})$ is introduced into the creep damage evolution equation. The creep damage evolution (Eq.(3)) can be expressed as,

$$
\dot{D}_{c}=\left(\frac{\|T\|-(1-D) T_{c}}{C}\right)^{r}(1-D)^{-P}\left(\vartheta-\vartheta_{0}\right)^{\xi}
$$

With $\xi$ as an additional model parameter for temperature influence. $\vartheta_{0}$ denotes the lower limit temperature below which the creep damage disappear.

\section{Verifications}

\subsection{Experiments}

The experiments were produced by Yang et al.[7,8]. The standard compact tension (CT) specimens (Fig.1(a)) with the width $\mathrm{W}=50 \mathrm{~mm}$, the initial crack length $\mathrm{a}=22.5 \mathrm{~mm}$ and the thickness $\quad B=25 \mathrm{~mm}$ were tested at $750^{\circ} \mathrm{C}$.

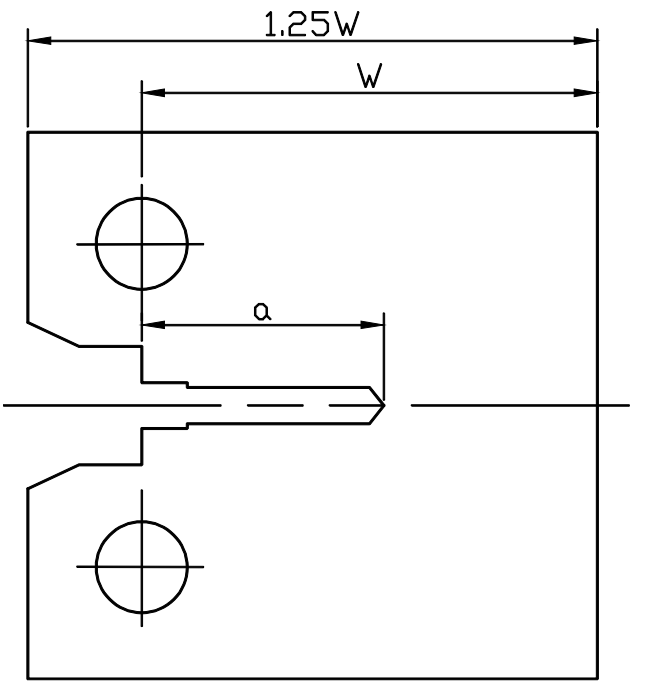

(a)

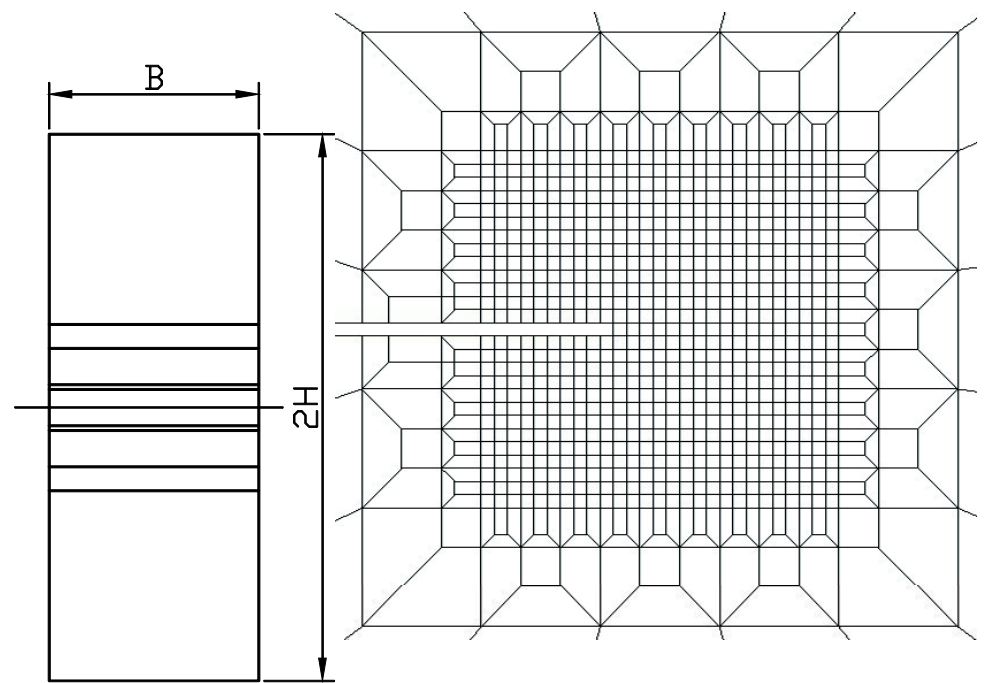

(b)

Fig. 1 Sketch of (a) compact tension (CT) specimen and (b) finite element mesh of crack tip.

Two wave shapes of applied loads were employed in the experiment, namely triangular and trapezoidal wave. The loading ratio was set to 0.05 . Two types of cycle loads contained $1.5 \mathrm{~s}$ loading and 1.5s unloading. Especially, the trapezoidal wave run with a 90s hold time at the maximum. Subsequently the hold time of 450s and 1500s were involved to consider the dwell time effects on fatigue crack growth.

\subsection{Results}

For the present material, the test results shown in Fig. 2 exhibit the effects of dwell time $\left(T_{d}\right)$ on the crack growth rate $d a / d N$ in terms of stress intensity factor range $\Delta \mathrm{K}$.

In Fig. 2 experiments with different dwell times are denoted using different symbols. Obviously, 
da/dN increases with $T_{d}$ in a power-law form. For large $T_{d}$ effects of dwell time diminishes. Computational predictions are shown in crosses with lines in the figure. The present cohesive zone model agrees roughly with experiments since both upper and lower limit cases are taken for parameter identifications. Increment of $d a / d N$ with dwell time, however, shows significant discrepancy between experiments and computations. Although the computations predict similar crack growth rate at large dwell time caused by the parameter identification for the cohesive zone model, the variations of the crack growth rate are very different. One may expect more significant deviations in prediction of the creep fatigue life with larger dwell time. The results imply that the damage evolution equation does not match the experimental observation accurately.

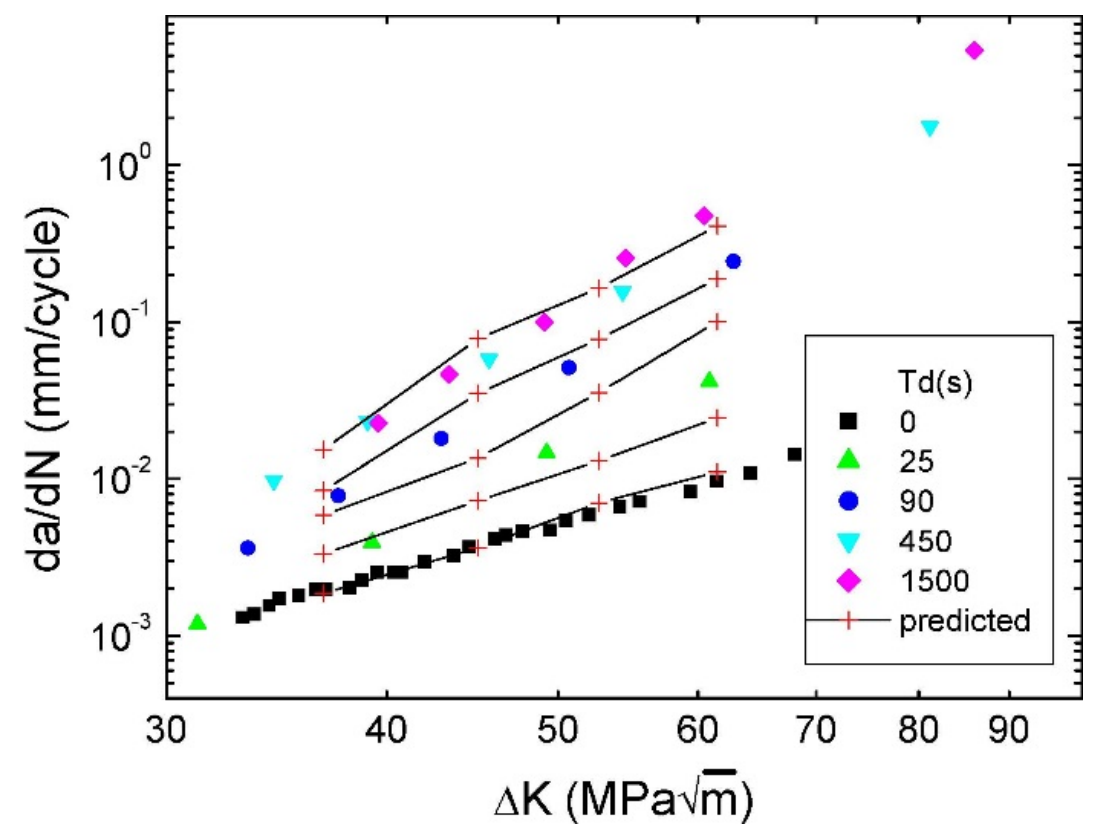

Fig. 2 Comparison of computational and experimental crack growth rates versus the stress intensity factor range $\Delta \mathrm{K}$ with different dwell times $\left(T_{d}\right)$ at $750^{\circ} \mathrm{C}$. The symbols denote experimental data and crossed lines stand for computational predictions.

\section{Conclusions}

Creep fatigue with long dwell times is generally difficult to be predicted. Using the cohesive zone model the present work has introduced a damage model for describing creep fatigue crack propagation with long dwell time for a nickel-based PM super-alloy. The computational results confirm:

1) The damage evolution equation suggests a nonlinear coupling of mechanical cyclic loading with temperature.

2) The cohesive zone model can predict the fatigue crack growth properly in the high frequency region. In fatigue with long dwell time the computational prediction still contains deviations.

3) The cohesive zone approach can be applied for more complex loading conditions or complex specimen geometries with great potential in numerical analysis of the creep-fatigue crack propagation.

\section{Acknowledgement}

In this paper, the research was sponsored by the National Natural Science Foundation of China (Project No. 51305025). 


\section{References}

[1] J. Tong, S. Dalby, J. Byrne, M. B. Henderson, and M. C. Hardy, "Creep, fatigue and xidation in crack growth in advanced nickel base superalloys," International Journal of Fatigue, vol. 23, no. 10, pp. 897-902, NOV 2001.

[2] Y. L. Lu, L. J. Chen, P. K. Liaw, G. Y. Wang, C. R. Brooks, S. A. Thompson, J. W. Blust, P. F. Browning, A. K. Bhattachary, J. M. Aurrecoechea, and D. L. Klarstrom, "Effects of temperature and hold time on creep-fatigue crack-growth behavior of HAYNES 230 alloy,” Materials Science and Engineering A-Structural Materials Properties Microstructure and Processing, vol. 429, no. 1-2, pp. 1-10, AUG 152006.

[3] G. I. Barenblatt, “The formation of equilibrium cracks during brittle fracture. General ideas and hypotheses. Axially-symmetric cracks,” Journal of Applied Mathematics and Mechanics, vol. 23, no. 3, pp. 622-636, 1959.

[4] Y. Xu, "Computational analysis of fretting fatigue,” Ph.D. dissertation, Bergische Universität Wuppertal, Jun 2009.

[5] K. L. Roe and T. Siegmund, "An irreversible cohesive zone model for interface fatigue crack growth simulation,” Engineering Fracture Mechanics, vol. 70, no. 2, pp. 209-232, 2003.

[6] J. L. Bouvard, J. L. Chaboche, F. Feyel, and F. Gallerneau, “A cohesive zone model for fatigue and creep-fatigue crack growth in single crystal superalloys,” Internatioanl Journal of Fatigue, vol. 31, no. 5, pp. 868-879, MAY 2009.

[7] H. Yang, R. Bao, J. Zhang, L. Peng, and B. Fei, "Crack growth behaviour of a nickel-based powder metallurgy superalloy under elevated temperature,” International Journal of Fatigue, vol. 33, no. 4, pp. 632-641, 2011.

[8] H. Yang, R. Bao, J. Zhang, L. Peng, and B. Fei, "Creep-fatigue crack growth behaviour of a nickel-based powder metallurgy superalloy under high temperature,” Engineering Failure Analysis, vol. 18, no. 3, pp. 1058-1066, 2011. 